rev.relac.int.estrateg.segur.5(2):109-136,2010

\title{
GLOBALIZACIÓN, RELACIONES INTERNACIONALES Y CRECIMIENTO ECONÓMICO*
}

\author{
Pedro Nel Páez Pérez** \\ Jorge Isaza Quebrada** \\ Luz Amanda Zamora***
}

\section{RESUMEN}

Este documento recoge los resultados preliminares del proyecto de investigación «Globalización, Relaciones Internacionales y Crecimiento Económico: una aproximación a América Latina», es el primer informe entregado como resultado de la investigación. Recoge básicamente el desarrollo del ciclo de la teoría económica y sus implicaciones para las relaciones internacionales aunque por ser un informe preliminar no profundiza en las relaciones entre la teoría económico, la globalización y las relaciones internacionales, más bien bosqueja algunas ideas de la forma en que se interrelacionan

* Este documento hace parte del Proyecto de Investigación EES 581 «Globalización, Crecimiento Económico y Relaciones Internacionales: Una Aproximación a América Latina», adscrito al Centro de Investigaciones de la Facultad de Relaciones Internacionales, Estrategia y Seguridad, de la Universidad Militar Nueva Granada.

** PhD en Economía, Director del Proyecto. Profesor, Escuela Superior de Administración Pública ESAP. pedronelpaez@gmail.com

${ }^{* * *}$ Co-investigador. Docente tiempo completo, Universidad Militar Nueva Granada. jorge.izasa@unimilitar.edu.co

${ }^{* * * *}$ Co-investigadora. luzamanda511@gmail.com. Se destaca la colaboración en este proyecto de dos estudiantes investigadores. 
estas variables pero son desarrolladas en profundidad en el texto final. La idea central considera que al igual que sucede con la actividad real, la recesión y la crisis económica implica un cambio en el paradigma teórico predominante de manera que teóricamente hablando, también puede hablarse de un ciclo de la teoría económica. El documento presenta, en forma concisa el análisis del desarrollo de la teoría económica desde el pensamiento mercantilista hasta la contrarrevolución de Chicago. A partir de esta revisión teórica se quiere mostrar posteriormente como la globalización, primero no es un fenómeno espontáneo; es más bien el fruto de un cuidadoso desarrollo teórico, especialmente a partir de los trabajos de Stigler y Friedman y segundo, la revisión teórica permite afrontar el problema de la globalización, el cambio en las relaciones internacionales y el crecimiento económico desde esa perspectiva histórico-teórica y que se manifiesta como un fenómeno multidisciplinar, complejo y que no se reduce simplemente al ámbito económico.

Palabras clave: Relaciones internacionales, teoría económica, globalización, crecimiento económico, geopolítica, cultura.

\begin{abstract}
This document presents the preliminary results of the research project «Globalization, International Relations and Economic Growth: An Approach to Latin America», and is the first product to be delivered as a result of the investigation. Basically reflects the development of the cycle of economic theory. As real activity, the recession and the economic crisis implies a change in the dominant theoretical paradigm. Also presents a concise analysis of the development of economic theory from mercantilism thinking to counterrevolution due to Chicago School of Thinking. From this theoretical review is to show how globalization, first is not a spontaneous phenomenon, is rather the result of a careful theoretical development, especially since the work of Friedman and Stigler and second, the theoretical review can address the problem of globalization, the shift in international relations and economic growth from the theoretical and historical perspective that manifests as a multidisciplinary phenomenon, complex and not simply reduced to the economic sphere.
\end{abstract}

Key words: International relations, economic theory, globalization, economic growth, geopolitics, culture.

\title{
INTRODUCCIÓN
}

Aun cuando la literatura sobre el ciclo económico es prolija, ver por ejemplo (David Backus, 1992), (Barro, 1977), (Basu, 1996), (Wen, 2003), (Robert Lucas, 1977) lo mismo no sucede con los análisis sobre el ciclo económico. Destacamos este hecho porque a partir del análisis del 
ciclo económico que desde luego se relaciona estrechamente con el ciclo real, pueden explicarse una serie de relaciones interesantes como la globalización y las relaciones internacionales. Este documento se enfoca en esa dirección y es el primer informe de un proyecto más ambicioso sobre globalización, crecimiento económico y relaciones internacionales, análisis que se aplicará al caso de América Latina. Está dedicado al estudio del ciclo de la teoría económica, bajo la creencia del equipo de trabajo que tal como ocurre con el comportamiento real de la economía, igual acontece con la teoría económica. Bajo la pretensión de ser un trabajo exhaustivamente teórico, en la primera parte se presenta una aproximación a la idea del ciclo teórico de la economía (Parte 2). Posteriormente se presenta un recorrido por los conceptos que dieron origen al pensamiento mercantilista y la fisiocracia (Partes 3 y 4). La Parte 5 hace una aproximación a la economía clásica. La Parte 6 está dedicada a la economía keynesiana; finalmente la Parte siete analiza la contrarrevolución de Chicago. Por último, se considera la relación entre crisis económica y cambio del paradigma teórico y se presentan unas conclusiones al trabajo.

\section{EL CICLO DE LA TEORÍA ECONÓMICA}

La historia humana muestra una relación entre el comportamiento real de la economía y la teoría económica, lo que sugiere la existencia de un ciclo teórico asociado con el ciclo económico. Las trasformaciones teóricas han estado precedidas por un comportamiento errático del desempeño económico que favorece el acomodamiento, la redefinición y el surgimiento de una teoría que reemplaza a la ortodoxia, aunque no necesariamente se trate de un cuerpo de conocimientos enteramente nuevo. Esta regularidad se ha advertido en periodos disímiles del tiempo en los que después de una gran crisis sobreviene una forma aparentemente «nueva» para explicar la realidad económica.

Las crisis económicas prolongadas inevitablemente cobran una cuota muy cara en materia social, pero comportan el replanteamiento y revisión de los paradigmas teóricos dominantes y la búsqueda de explicaciones alternativas, se buscan respuestas para los tiempos difíciles que no se encuentran en el armazón teórico vigente.

El ciclo de la teoría económica alcanza el punto más bajo cuando la corriente principal no permite, con las herramientas disponibles, explicar la crisis económica y el malestar social en un momento histórico específico. El desastre económico se lleva consigo también unos paradigmas, unas creencias, unas formas de ver el mundo y se inicia el ascenso de otras ideas, otras concepciones contrapuestas que en principio suponen la superación de la crisis, el aumento del bienestar y la cima para un conjunto de conceptos si no enteramente nuevos, si actualizados de acuerdo a las circunstancias determinadas y el desarrollo continuo de la ciencia. Particularmente en la economía una estructura teórica que pase a un segundo plano no desaparece, dejará de ser la corriente principal desde la cual se pueden sustentar algunos fenómenos sociales como el crecimiento, el empleo, el nivel de precios, la inflación, pero está en continuo desarrollo pues en una 
coyuntura severa como una nueva crisis la convierte probablemente de nuevo en el paradigma dominante en detrimento del existente, y llega a constituirse en nuevamente en ortodoxia. Y nos parece advertir un movimiento pro-cíclico de la teoría con respecto al producto. Tasas positivas de crecimiento de los indicadores económicos se relacionan positivamente con el afianzamiento de una corriente teórica en detrimento de otra, pero el cambio de signo de los indicadores indica también el cuestionamiento de su validez, de su solidez, e inicia su parte descendente del ciclo (igual que el producto) pero al mismo tiempo cobran fuerza otro conjunto de ideas, de conceptos. A partir de las prescripciones de política se recupera el crecimiento y la consolidación de estas ideas «nuevas» hasta que alcanza la cima y una nueva crisis las someta al debate, a la validación de los supuestos, al cuestionamiento.

En torno a las consideraciones anteriores deben evitarse las generalizaciones radicales. No se observa el ascenso de una teoría nueva cada vez que sobreviene una crisis pero sí dependerá en gran medida de la severidad y extensión, temporal y geográfica de ésta. Incluso se puede alegar, razonablemente, el surgimiento de teorías totalmente nuevas porque coexisten en un tiempo continuo con diferentes grados de desarrollo y avances dinámicos innegables que se registran dentro unos marcos de referencia más amplios. Por ejemplo, la revolución keynesiana se sintetizó en el famoso debate «Keynes versus los clásicos» y a pesar de los elementos nuevos del keynesianismo éste se construye retomando algunos elementos esenciales del mercantilismo y contribuciones relacionadas hasta llegar a ser el paradigma explicativo dominante y el debate «neoclásicos versus Keynes» retoma elementos fundamentales de la economía clásica, sin duda con elementos novedosos hasta constituirse asimismo en una corriente dominante.

En la conformación histórica del ciclo de la teoría económica hay dos elementos asociados con la génesis de los fundamentos teóricos de la economía que influyeron también en la configuración de dos tendencias importantes o escuelas de pensamiento, incluso dos corrientes teóricas principales que curiosamente se suceden en el tiempo. Se trata del grado de apertura de la economía y el papel del Estado en la actividad económica ${ }^{1}$. En torno a de estos principios generales se establecieron las vertientes teóricas que han predominado hasta hoy pero hay que ver la forma en que cada uno de esos principios se articula en cada teoría. Una vertiente es partidaria de la apertura económica y un papel muy reducido del estado en la gestión económica. La apertura se sustenta en la libertad de mercado (interno y externo) la flexibilidad de precios y el ajuste automático de la oferta y la demanda de bienes y servicios alrededor de un punto de equilibrio. Esta posición se asocia con la fisiocracia, la economía clásica, la revolución de las expectativas racionales, el monetarismo e ideológicamente cercana al neoliberalismo. La otra vertiente considera necesaria la intervención pública en los asuntos económicos y sociales, la protección de la economía local por las restricciones al comercio internacional, la regulación de

1 Estas concepciones no son mutuamente excluyentes, por supuesto, pues en algunos puntos se traslapan y aún en otros no llegan a ser radicalmente diferentes. 
algunos mercados con un alto grado de discrecionalidad pública en el diseño, planeación y ejecución de la política económica junto con altos grados de regulación (normatividad) al sector privado. La intervención y la regulación suponen la rigidez de algunos precios, como el tipo de cambio y los salarios. Se asocia comúnmente con la economía keynesiana, el estado del bienestar, la economía pos-keynesiana, la planificación económica y el intervencionismo. Justo es reconocer que hacer generalizaciones puede inducir un reduccionismo teórico en el sentido en que de acuerdo a lo expresado, la teoría económica se reduce a dos escuelas o dos teorías solamente. El inconvenientes se supera si se reconoce que estas dos corrientes se han complejizado de diversas maneras y derivado en amplias ramificaciones, pero como origen común tiene los elementos básicos sobre la apertura y la intervención.

El ciclo de la teoría económica se expresa también en un modelo económico concreto. La teoría es el soporte del modelo, porque no se concibe un modelo sin teoría lo mismo que no existe una teoría sin que sus principios se traten de aplicar a la realidad por medio de un modelo abstracto, junto con las implicaciones económicas, políticas, sociales y culturales que conlleva la adopción de uno u otro modelo. Por esa razón no es raro oír hablar de crisis económica y cambio en el modelo de desarrollo. En lo que sigue, se tratará de resaltar los elementos más importantes de estas vertientes teóricas de la economía desde sus orígenes y hallar una relación con las relaciones internacionales, entendiendo que cada vertiente tiene una configuración para las relaciones internacionales.

\section{EL MERCANTILISMO}

El sistema mercantilista perduró a lo largo de 300 años, desde finales del siglo XV hasta el siglo XVIII como lo destaca Mills (Mills, 2003) y se desarrolla a la par de una serie de acontecimientos trascendentales para la época, como el descubrimiento de América en 1492 y las rutas comerciales que unieron a Europa con África. El ensanchamiento del mundo conocido marca el inicio de la época colonial y la conformación de un orden internacional expansionista al tiempo que se configura el estado-nación, la lucha entre estados por los metales preciosos y el monopolio exportador, orden internacional marcado por las relaciones de violencia en la explotación de los recursos físicos y humanos de las colonias. Políticamente se consolida un estado nacional por encima del individuo y la empresa, estamental y burocrático, que a la vez que articula los diferentes estamentos y permite la acumulación de capital determina un orden interno dado poder eventualmente ilimitado de los gobernantes. En el ámbito externo, el mercantilismo promueve el comercio como un medio de obtener riqueza y poder a través del privilegio de la capacidad exportadora sobre la importadora, el control de la actividad económica y la dominación de los mercados periféricos como proveedores de materia prima y consumidores de manufacturas. Ello permite una acumulación de capital cuyo destino es el progreso y la acumulación de poder estatal y uen la perspectiva de la teoría de las relaciones internacionales, la práctica del mercantilismo supone 
la adopción de las premisas del realismo clásico que entiende la historia como testimonio del conflicto, el contexto externo como esencialmente anárquico, el interés nacional como traducción del poder y el escenario económico como sometido al político dominado por el Estado. Si el ejercicio de la guerra es natural a este orden de cosas y el poder depende en buena cuenta del predominio comercial, el comercio exterior es una función de la seguridad nacional... la práctica y la racionalización del mercantilismo tiene múltiples facetas (que van desde la expansión imperial hasta la extrema protección del mercado nacional) y muy variados escenarios de aplicación».

La consolidación y fortalecimiento del estado-nación influye decisivamente en la creación del mercado interno y una forma antigua pero fuerte de intervencionismo para regular el comercio externo organizado en el monopolio exportador y la restricción a las importaciones por el control de los tipos de cambio. Estas medidas indujeron el desarrollo de la industria nacional especialmente europea ya que permitía elaborar los bienes destinados a la exportación a las colonias, en la mayoría de los casos.

Igualmente consideraron a la balanza comercial superavitaria como una fuente importante de la prosperidad nacional, el superávit permite acumular metales preciosos (Shumpeter, 1995) y generar un excedente producido en el monopolio exportador; en consecuencia, las reservas de metales preciosos se asociaron con la riqueza, aunque no previeran sus efectos inflacionarios. Se creía que el aumento de la cantidad de dinero no tenía efectos inflacionarios dado que el volumen de transacciones comerciales se amplía, pari passu, con el dinero extra que está disponible.

La primera parte del ciclo en el pensamiento económico alcanza un apogeo con los pensadores mercantilistas, pero suscita un conflicto inicial con la fisiocracia, que terminará favoreciendo el replanteamiento de los principales argumentos mercantilistas ante la situacion económica y social del siglo XVI, marcada por la crisis y las dificultades. El mercantilismo pasará a un segundo plano como un intento de interpretar la realidad económica. A partir de los supuestos de la fisiocracia surje algo difuso, rudimentario, que se consolidará y define algunos de los elementos que posterormente dan forma a un paradigma que durará bastante tiempo, la economía clásica. A un nivel más general se está dando el cambio de una época a otra, una transición del feudalismo al capitalismo, el crecimiento demográfico, la escacez los metales preciosos, que afectan especialmetne al comercio y que crean las condiciones para la expansión de la manufactura, las relaciones salariales y un enfoque novedoso para abordar el estudio de la economía.

\section{LA FISIOCRACIA}

Se atribuye su origen a un grupo de pensadores franceses posteriores a la revolución. Para Mills (op. cit. 2003:55 y ss.) «fueron los primeros en desarrollar una descripción del proceso económi- 
co como un todo, empleando métodos científicos de aislamiento y abstracción» y como señala Bloomfield, el mercantilismo, para le época de los fisiócratas «mostraba signos definitivos de desintegración» (Bloomfield, 1938) y nace justamente como una reacción al pensamiento mercantilista y las regulaciones al comercio externo. En consecuencia, defienden una disposición al libre comercio y la intervención mínima del gobierno en la economía. Ver por ejemplo (E. Screpanti, 1997) para quienes «el mercantilismo, un planteamiento teórico relativamente homogéneo que había dominado el pensamiento económico europeo durante 300 años, creando así una comunidad científica internacional, se vio súbitamente atacado desde diversos frentes, y en el transcurso de un cuarto de siglo desapareció de la escena económica» Screpanti. et. al. 1997).

La fisiocracia elabora una representación esquemática de la economía por medio de la tableau economique, cuya autoría se atribuye a Quesnay (Quesnay, 1758 (1972)). La economía es un flujo circular de la renta, se reproduce anualmente hacia posiciones de equilibrio, concepto que será muy importante en el análisis económico posterior. Para el siglo XVII, la agricultura y los dueños de la tierra tienen la primacía económica y social en Francia de manera que los fisiócratas asocian la riqueza con la posesión de tierras y las actividades agrícolas, ya que permiten obtener el producto neto o valor agregado de la producción; en esa concepción, los comerciantes y los industriales se tienen por clases estériles porque se apropian de una parte del producto neto obtenido en la agricultura sin generar ningún valor agregado en el proceso. Los fisiócratas promueven la actividad agrícola y a diferencia del mercantilismo, son partidarios de eliminar las regulaciones al comercio internacional y una intervención mínima del estado en la gestión económica, principio que será conocido posteriormente como laissez-faire.

Respecto al comercio externo, supusieron que el aumento de las exportaciones de productos agrícolas, en lugar de manufacturas, favorecía el interés nacional; si sus precios eran altos, se generarían los recursos para que la clase estéril exportara sus productos más allá de las fronteras nacionales. Cabe anotar que algunos conceptos concebidos por los fisiócratas se convirtieron más tarde en aportes para la economía clásica. Entre ellos se encuentran el valor de la tierra como factor productivo, el flujo circular de la renta, la ley de Say, la oposición a la intervención pública y el principio mismo de laissez faire. En resumen, replantearon algunos principios mercantilistas, sistematizaron los propios con los que se construye posteriormente la teoría económica en sus inicios.

\section{LA ECONOMÍA CLÁSICA}

La economía clásica tiene se fundamenta en el liberalismo filosófico especialmente de Hobbes (Hobbes, 2009) y Locke, (Locke, 2003) el individualismo metodológico, el principio del laissez faire, la competencia perfecta y el utilitarismo, asociado con Benthan (Bentham, 2007) y J. S. 
Mill (J S Mill, 2009). El estado se concibe como garante de los derechos de propiedad y los derechos individuales, especialmente la propiedad privada. A diferencia del comerciante mercantilista y el señor de la tierra de la fisiocracia, la economía clásica recalca el papel del empresario afín con la revolución industrial y desde luego el desarrollo de esta vertiente del pensamiento económico se beneficia por el desarrollo tecnológico y el método científico. Por otra parte, la economía clásica (como en alguna medida lo fue la fisiocracia) es un raciocinio en contra de la primera ortodoxia dominante en la época, el mercantilismo y al igual que los fisiócratas, refutaron el bullionismo o la creencia según la cual la acumulación de metales preciosos constituía una fuente de riqueza.

La economía clásica se divide en dos fases. La primera, a la que pertenecen Adam Smith, (Smith, 1776), David Ricardo (Ricardo, 2010) y J.S. Mill (John Suart Mill, 2004) se fundamenta en la teoría del valor trabajo, el principio de utilidad marginal decreciente y la utilidad cardinal; la segunda, por la teoría del valor utilidad y la utilidad ordinal, llamada también revolución neoclásica, donde se destacan figuras como Menger (Menger, 2007), Marshall, (Marshall, 2009) Pigou (Pigou, 2010) y Walras (Walras, 2003). Si bien es evidente un cambio fundamental en algunos de los principios esenciales, es en principio la misma teoría (Ikeda, 2006) (pp. 38) construida en torno a la eficiencia del mercado y desprovista de las distorsiones e inflexibilidades introducidas por las diversas formas de intervención pública.

Con la publicación de la Riqueza de las Naciones se inicia la construcción sistemática de una estructura teórica apuntalada en el teorema de la mano invisible y el principio de laissez-faire. Las decisiones de los agentes económicos liberadas de la acción pública son el fundamento de la libertad de mercado y del bienestar social. La base material del bienestar es la producción y consumo libre de bienes y servicios por los agentes económicos y las decisiones autónomas que éstos toman. La acción pública se circunscribe al cumplimiento de algunas tareas esenciales que apoyan y complementan el buen funcionamiento del mercado como la defensa nacional, el orden público y la recaudación de impuestos. (Al respecto ver A. Smith (op. cit.) libro quinto sobre los gastos e ingresos del soberano o de la República).

La búsqueda del interés propio por los consumidores (demanda) y los empresarios (oferta) liberados de las restricciones que impone el gobierno conforman el núcleo de la economía clásica, como afirma Smith «...el hombre reclama en la mayor parte de las circunstancias la ayuda de sus semejantes y en vano puede esperarla sólo de su benevolencia. La conseguirá con mayor seguridad interesando en su favor el egoísmo de los otros y haciéndoles ver que es ventajoso para ellos hacer lo que se les pide» (Smith, ibid.:17). La oposición a la intervención pública en los períodos de desempleo temporal imposibilita el ajuste y el equilibrio, menoscabando aún más la situación económica. Si por el contrario, no hay interferencias, la acción de la mano invisible de los mercados en los períodos de desempleo transitorio permite que la economía se auto-ajustarse hasta restaurarse nuevamente el equilibrio. 


\subsection{Los axiomas de la economía clásica}

\subsubsection{La Ley de Say}

La Ley de Say (Say, 1803), conocida ya para el siglo XIX, es un principio esencial del argumento clásico; un mercado competitivo con salarios y precios flexibles opera como un mecanismo que asegura que las fuerzas del mercado (oferta y demanda) favorecen unas condiciones económicas en las que todos los recursos disponibles se emplean completamente. Se atribuye a Mill el haber acuñado el término por el que se conoce esta Ley, al aseverar que la oferta crea su propia demanda y desde entonces se ha popularizado bajo esa acepción.

Los individuos perciben que el trabajo genera des-utilidad al tenerse por una actividad poco placentera, pero proporciona los ingresos para comprar bienes y servicios producidos por la industria y al contrario del trabajo, el consumo proporciona placer. En ese orden de ideas, la gente trabaja si obtiene los ingresos suficientes para comprar bienes y obtener utilidad, pero en la medida que compense la des-utilidad que representa el trabajo de obtener los ingresos, de manera que «todos los trabajadores detentores de renta no estarían maximizando su bienestar económico si se comprometen en el acto desagradable de contribuir en la producción para obtener el ingreso, si no intentaran gastar cada peso que ganan en el placer que obtienen de los bienes y servicios producidos por la industria.» (Davidson, 2009).

Si los individuos son egoístas y racionales², la renta recibida en el mercado por el factor trabajo en razón de la producción y venta de bienes y servicios se debe gastar para demandar la producción industrial. Es lógico entonces que no haya nunca una escasez de demanda para todos los bienes que puede producir la economía cuando emplea plenamente sus recursos.

\subsubsection{La neutralidad del dinero}

El supuesto sobre la neutralidad del dinero es una de las piedras angulares de la teoría económica clásica, pero también una materia de fe subyacente, tanto para la revolución neoclásica de finales del siglo XIX como para la teoría económica dominante actual o mainstream ${ }^{3}$. «...hoy en día se mantiene como uno de los axiomas fundamentales de la corriente principal en la teoría económica ... es un artículo de fe que no requiere ni ser probado ni justificado». (op. cit. Davidson: 41). El axioma sugiere que las fluctuaciones en la cantidad de dinero no tienen efectos sobre los niveles de producción y empleo; por el contrario, estas variables en el agregado estarían determinadas solo por factores no monetarios inherentes al sistema económico.

2 Aquí la racionalidad debe interpretarse en el sentido moderno, como agentes que maximizan sus respectivas funciones objetivo, bien sean consumidores o productores.

3 En la teoría del ciclo económico, muchos de los postulados clásicos van a retomarse por el «fundamentalismo» de Chicago, como se verá más adelante. 
En la teoría clásica los bienes y servicios se intercambian por bienes y servicios. Puesto que la oferta siempre es igual a la demanda (por la ley de Say) el dinero solo es necesario como un medio contable, un numerario en el proceso de producción e intercambio. Se trata en esencia, de una economía de trueque, en la que las transacciones se realizan cuando el poseedor de una mercancía intercambia una cantidad específica de un bien por una cantidad específica de otro; proceso que puede llegar a ser bastante dispendioso dado el número amplio de bienes producidos por la industria. En una situación como esta, y para la teoría clásica, el dinero es solamente un medio que facilita la contabilidad de los intercambios de trueque.

\subsubsection{La sustitución bruta}

La existencia de sustitutos brutos significa que todo bien es un buen sustituto de todos los demás bienes y el cambio en los precios relativos induce a los compradores a demandar los bienes que son relativamente más baratos y menos de aquellos que se han encarecido, gastando la misma cantidad de dinero. El axioma asegura que, si los precios en el mercado son perfectamente flexibles, cuando a los precios vigentes no se venden todos los bienes producidos, los fabricantes siempre pueden realizar los inventarios bajando los precios de mercado en relación con los demás precios.

Por analogía, se sigue que si a cualquier tasa salarial dada hay trabajadores desempleados, y dado el axioma de sustitutos brutos, todos los trabajadores serán contratados sólo si el salario vigente en el mercado se reduce. Si los bienes producidos se venden a los precios vigentes, se dice que los mercados se «despejan», de manera que el pleno empleo ocurre cuando el mercado de trabajo se despeja. Cuando todo aquel que quiere trabajar a los salarios vigentes en el mercado tiene un trabajo, no hay desempleo involuntario. «Los trabajadores están empleados involuntariamente si, en el evento de un pequeño incremento de los precios de los bienessalario relativo al salario nominal, tanto la oferta agregada de trabajo dispuesta a trabajar por el salario nominal corriente y la demanda agregada de trabajo a ese salario es mayor al volumen de empleo (Keynes, 1936).

\subsubsection{La incertidumbre y el axioma ergódico}

En épocas pasadas los economistas creían que tenían información completa sobre el pasado y el presente y por tanto sobre el futuro y con base en eso, tomaban decisiones correctas sobre el futuro, por lo que en un mercado libre, los participantes toman las mejores decisiones que representan mejor sus intereses. El futuro siempre se conoce y parece ser próspero.

Si la senda temporal de una economía y su comportamiento futuro es el resultado de un proceso estocástico, el futuro de cualquier decisión actual estaría determinado por una distribución probabilística (ibíd.:30), de manera que para efectuar predicciones probabilísticas confiables sobre un resultado económico futuro, el tomador de decisiones recopila y analizar datos muestrales 
sobre el futuro para estimar, con un alto grado de confianza estadística, el valor futuro de mercado de ese resultado. Como no es posible obtener datos muestrales del futuro que permitan afirmar que el estado de la economía se determina por un proceso estocástico, se opta por muestras de datos pasados y actuales, para aseverar que estos datos equivalen a tomar una muestra del futuro, es decir, el axioma implica que el resultado en cualquier fecha futura es la «sombra» estadística de los datos pasados y presentes y «el cálculo de probabilidades entrelaza el presente y el futuro en el modelo clásico, argumentando que el futuro será como el presente y que las consecuencias de cualquier cambio futuro se pueden evaluar fácilmente» (Sheehan, 2009).

La realización de un proceso estocástico se define como el valor muestral de una variable multidimensional en un periodo de tiempo, por ejemplo, una serie temporal única de algunos resultados observados, así, el proceso estocástico proporciona un universo de esas series temporales. Las estadísticas temporales se refieren a promedios estadísticos calculados desde realizaciones singulares en un período de tiempo calendario y las estadísticas espaciales se refieren a promedios estadísticos calculados en un punto fijo del tiempo y se forman sobre el universo de las realizaciones. Se calculan a partir de datos de corte transversal, es decir, datos obtenidos de participantes individuales en un solo punto del tiempo.

Entonces sí, y sólo sí, el proceso estocástico es ergódico, para realizaciones infinitamente grandes las estadísticas espaciales y temporales coinciden. Para realizaciones finitas de los procesos ergódicos, las estadísticas espaciales y temporales coinciden excepto para los términos de error estocástico. En otras palabras, calcular las estadísticas espaciales y temporales tiende a converger (con una probabilidad de uno) cuando el número de observaciones se incrementa. Consecuentemente, si el axioma ergódico es aplicable, las estadísticas calculadas o bien por series de tiempo pasadas o datos de corte transversal son estadísticamente estimaciones confiables del espacio de estadísticas que puede ocurrir en cualquier fecha futura.

El axioma asegura que el resultado asociado con cualquier fecha futura puede predecirse con seguridad por medio del análisis estadístico de los datos ya existentes obtenidos o bien de las series de tiempo o de datos de corte transversal. Así el futuro nunca es incierto y siempre puede ser predicho con seguridad por medio de un análisis estadístico suficiente de datos ya existentes, es decir, no existe incertidumbre. Los resultados futuros, en el sistema ergódico, son probabilísticamente riesgosos pero predecibles con toda seguridad ${ }^{4}$.

4 De acuerdo con Davidson (op. cit.) en los modelos económicos clásicos no probabilísticos el axioma de completitud de la teoría clásica juega el mismo papel del axioma ergódico. Este axioma asume que en cualquier punto del tiempo la gente «conoce» todos los resultados futuros posibles de cualquier acción tomada hoy y puede ordenar correctamente estos resultados posibles asociados con varias elecciones en una lista desde perspectivas más preferidas a menos. 
En esencia, el axioma ergódico afirma que el futuro siempre puede predecirse estadísticamente con seguridad calculando las probabilidades de datos del mercado presentes y futuros y aplicar estas probabilidades a resultados futuros posibles. Presume que un resultado futuro de cualquier decisión hecha hoy, puede predecirse con un alto grado de precisión estadística.

\subsection{Determinación del nivel de producción y empleo}

Según la proposición clásica de neutralidad del dinero, el nivel de producción real es independiente de la cantidad de dinero y se determina por la función de producción de corto plazo de la forma $Y=A F(K, L)$.

Esta función expresa la cantidad máxima de producto que puede obtenerse con cantidades fijas de factores productivos. Ya que en el corto plazo la productividad del trabajo (A) y el capital (K) permanecen constantes, el factor variable es el trabajo, lo que equivale a decir que en el corto plazo la producción agregada se modifica únicamente por las variaciones de la cantidad de trabajo utilizado.

Dados $(A)$ y $(K)$, la relación entre los niveles de producción y empleo es positiva; la productividad marginal del trabajo tiene un efecto positivo sobre la producción. Cuando el trabajo aumenta en una unidad, la producción también aumenta; hay un desplazamiento sobre la función de producción; pero cada vez menor por el supuesto de rendimientos constantes a escala y en un punto la productividad marginal del trabajo es decreciente, es decir, $P M g_{L}<0$. Mejoras en la tecnología y en el capital, como en el caso de innovaciones o descubrimientos científicos aplicados al proceso productivo, caso que era muy frecuente desde la revolución industrial así como aumentos en el stock de capital, desplazan la función de producción en sentido ascendente.

Para determinar el nivel agregado de empleo debe considerarse el mercado laboral clásico, que cumple la ley de Say. Así puede establecerse la cantidad de trabajo necesario para alcanzar un determinado nivel de producción. Suponiendo una estructura de mercado de competencia perfecta, la condición de maximización de beneficios para las empresas está dada por la igualdad entre el ingreso marginal, IMg y el costo marginal Cmg; por lo que, dada la estructura de mercado, $I M g=C M g=P$, luego entonces,$P=C M g$.

Las empresas contratan trabajo en un mercado laboral competitivo en el que, a cada trabajador adicional que ocupen se paga un salario nominal $W$. El costo marginal está dado por $W \Delta L$ que aumenta la producción en $\Delta Q$, que al multiplicarse por el precio $P$ produce el ingreso adicional $P \Delta Q$ o ingreso marginal. La economía emplea adicional trabajo si $W L<P \Delta Q$. Y la maximización del beneficio se cumple cuando $P \Delta Q=W \Delta L$ :

$$
\frac{\Delta Q}{\Delta L}=\frac{W}{P}
$$


La productividad marginal del trabajo es igual al salario real. De la anterior expresión se deduce que

$$
C M g=\frac{W}{P M g_{L}}
$$

Por otra parte, $P \Delta Q=W \Delta L$. Estas dos últimas expresiones muestran la condición de maximización de beneficios; el ingreso marginal es igual al costo marginal. Puesto que el beneficio se maximiza cuando,

$$
P M g_{L}=\frac{W}{P}
$$

la curva de producto marginal es equivalente a la curva de demanda de trabajo, por lo que

$$
D_{L}=D_{L}\left(\frac{W}{P}\right)
$$

De lo anterior se cumple que como era de esperarse, en el agregado, la demanda de trabajo clásica por las empresas es una función inversa del salario real: a menor salario real se demanda más trabajo y viceversa.

De otra parte, la oferta de trabajo clásica está dada por $S_{L}=S_{L}\left(\frac{W}{P}\right)$

Asumiendo el supuesto de que las familias maximizan la utilidad, la oferta agregada de trabajo es una función creciente del salario real y depende también de las preferencias de la fuerza laboral entre ocio y consumo, pues ambos reportan una utilidad positiva, pero el consumo procede de la dicotomía entre ocio y trabajo y la renta necesaria para el consumo. Dado que el trabajo implica des-utilidad, las preferencias de la fuerza laboral entre ocio y consumo y el salario real determinan la oferta de trabajo de equilibrio.

Un incremento del salario real produce un efecto sustitución, ya que se encarece el ocio y aumenta la oferta de trabajo, pero al mismo tiempo, ante un salario real mayor, mejora la situación de los trabajadores y por tanto quisieran disfrutar de mayor ocio, produciendo un efecto renta. Si, como suponían los economistas clásicos, el efecto sustitución domina al efecto renta, la oferta de trabajo responde positivamente a un aumento del salario real. (Ver Snowdon, op. cit: 50).

\subsection{Producción de equilibrio y nivel de empleo}

El mercado laboral clásico se despeja en el punto donde se igualan la demanda y la oferta de trabajo y se establece un salario real $\frac{W}{P}$ con un nivel de empleo de equilibrio $L$. Si el salario real es menor al salario real de equilibrio, se produce un exceso de demanda de trabajo y en consecuencia un aumento del salario real como respuesta a la mayor demanda de trabajo por las empresas (demandan más trabajo) hasta restaurar el salario real de equilibrio; si el salario real es 
mayor al salario real de equilibrio, ocurre un exceso de oferta de trabajo que induce a la baja los salarios reales hasta que se alcance de nuevo al salario de equilibrio, garantizado por una estructura competitiva en el mercado laboral, precios flexibles e información completa.

El nivel de empleo alcanzado en el mercado de trabajo competitivo clásico es de pleno empleo; todos los miembros de la fuerza laboral que desean trabajar pueden hacerlo al salario real de equilibrio vigente, determinado en este mercado y admite la existencia de desempleo friccional y voluntario, pero no la posibilidad de desempleo involuntario. El concepto de desempleo friccional se relaciona con la pérdida de tiempo cuando se busca un trabajo y el desempleo voluntario cuando un trabajador rehúsa aceptar un trabajo por una remuneración correspondiente al valor atribuible a su productividad marginal (Viner, 1936).

Con los elementos expuestos en los párrafos anteriores, en el modelo clásico se explicaron el nivel de producción real de equilibrio, el nivel de empleo de equilibrio, así como los salarios reales y el nivel de desempleo de equilibrio. La competencia en el mercado de trabajo asegura la existencia de pleno empleo, de manera que cualquier distorsión o inflexibilidad introducida en el mercado de trabajo que eleve los salarios reales de equilibrio, como la existencia de legislación sobre salario mínimo o la organización de los trabajadores en sindicatos (aquellas variables institucionales en la famosa $z$ de la función de producción agregada de Blanchard) desde los que puedan presionar salarios mayores, se refleja en un salario real mayor al de equilibrio y distorsionado, ocasionando que haya gente que no puede trabajar. La solución al desempleo clásico consistió en reducir los salarios reales recortando el salario nominal.

\subsection{El tipo de interés}

Los clásicos asumieron que el ahorro se incrementa cuando existen excesos de efectivo y dinero ocioso si el tipo de interés es positivo. Ante un aumento del tipo de interés el ahorro se mueve en la misma dirección, en consecuencia, el interés actúa como determinante en las decisiones entre ahorro y consumo. A mayor tipo de interés, mayor ahorro.

El tipo de interés es significativo en tanto asegura que no hay insuficiencia de demanda agregada. Dada una economía de dos sectores, firma y hogares, $E=C(r)+I(r)=Y$ representa el gasto agregado que es igual a la producción y donde el consumo, $C$, como el gasto en inversión $I$, son funciones del tipo de interés $r$. La demanda planeada de bienes es igual a la suma de la demanda planeada por bienes de inversión, como función del tipo de interés, $r$. Puesto que los hogares no gastan toda su renta, $Y-C(r)=S(r)=I(r)$. El ahorro es, como el consumo, en el sistema clásico, una función del tipo de interés, $r$. Ante incrementos del tipo de interés habrá un mayor nivel de ahorro y mayor consumo futuro. El interés actúa como una recompensa real por postergar el consumo presente. El ahorro representa una oferta de fondos prestables en el mercado de capitales. Dado que $\frac{\partial S}{\partial r}>0$, el consumo se relaciona nega- 
tivamente con el tipo de interés, es decir $\frac{\partial C}{\partial r}<0$. Por su parte, el gasto en inversión se relaciona negativamente con el tipo de interés, o $\frac{\partial I}{\partial r}<0$ o demanda de fondos prestables en el mercado de capitales. La inversión se realiza si la tasa esperada de retorno es mayor o igual al costo de acceder a los fondos prestables utilizados en la compra de los bienes de capital. Con un aumento el tipo de interés, se aumentan los costos.

\subsection{Inversión, ahorro y tipo de interés}

La productividad y el ahorro determinan el tipo de interés real y sus variaciones equilibran el mercado de fondos prestables. La tasa de interés es flexible y cambia para igualar los deseos de ahorrar e invertir y también opera, para el mercado de dinero, la ley de Say. Los salarios, los precios y los tipos de interés son flexibles.

La operación de los mercados de capitales y trabajo refuerza la ley de Say y proporciona a los economistas clásicos un sistema teórico capaz de explicar la determinación de las variables reales dentro del sistema. La separación de las variables reales de las variables nominales se denomina la dicotomía clásica y les permite examinar el comportamiento de las variables reales en el sistema económico omitiendo las variables nominales, por lo que la cantidad de dinero no juega ningún papel en la determinación de las variables reales, de manera que la neutralidad del dinero en el largo plazo es otra característica importante en el desarrollo de esta estructura teórica. La teoría cuantitativa del dinero permite a los clásicos explicar la determinación de las variables nominales, o teoría monetaria que tuvo vigencia hasta 1930 y se asocia con Marshall y Pigou y la ecuación de Cambridge y Fisher.

La versión marshalliana de la teoría cuantitativa del dinero se expresa por la ecuación de Cambridge. Establece una diferencia entre la demanda de dinero, $M^{d}$ y la oferta de dinero $M^{s}$. La demanda está determinada por la necesidad de realizar transacciones y mantiene una relación positiva con el valor nominal del gasto agregado que a su vez es igual a la renta nominal, por lo que $M^{d}=k P Y^{d}$ donde $M^{d}$ es la demanda por saldos monetarios reales y $k$ es una fracción de la renta nominal nacional por periodo. Esta expresión es la ecuación de Cambridge, que en términos clásicos representa la demanda de dinero. Si por la acción de la autoridad monetaria la oferta monetaria es constante, entonces se tiene que $M^{s}=M^{d}$ y representa el equilibrio en el mercado de dinero, por lo que $M^{s}=k P Y^{d}$.

Puesto que $Y$ está predeterminado en su nivel de pleno empleo de equilibrio por la función de producción de corto plazo, con pleno empleo y un mercado de trabajo competitivo, la cantidad de dinero no afecta las variables reales en el largo plazo, pero sí determina el nivel de precios. Asumiendo que $Y$ y $k$ son exógenos, la oferta de dinero determina el nivel de precios. Si el mercado de dinero está en equilibrio cuando $M^{d}=M^{s}$ y se considera un aumento de la cantidad 
de dinero, se crea una situación de desequilibrio con $Y, k$ constantes y sólo se puede restaurar este equilibrio si aumenta el nivel de precios. La lógica que impera para los clásicos es que los agentes económicos al tener más dinero del que desean tener, ese saldo de exceso de dinero que se presenta por el aumento de la cantidad de dinero lo utilizan para comprar más bienes y servicios y puesto que la oferta de bienes y servicios está restringida por el nivel de producción (de equilibrio), este exceso de demanda causa el incremento en el nivel de precios, proporcionalmente al aumento inicial en la oferta monetaria.

La ecuación de Fisher, para explicar la teoría cuantitativa del dinero, o ecuación de intercambio, está dada por $M V=P Y$.

Donde $V$ es la velocidad del dinero y se define como el recíproco de $k$.

$$
V=\frac{1}{k}
$$

Siendo $V, P$ constantes, puede verse que $P$ depende de $M$ y $\Delta M=\Delta P$. Las variables reales, como la producción y el empleo no se ven afectadas por la cantidad de dinero. El mercado de trabajo, que es competitivo, genera un nivel de empleo de equilibrio, por decirlo $L_{0}$ y un salario real $\frac{W_{0}}{P_{0}}$ compatibles con un nivel de producción $Y_{0}$. Dado que la curva de oferta de trabajo clásica es perfectamente inelástica, el nivel de producción no varía con el nivel general de precios. La curva de demanda agregada clásica se obtiene de la ecuación $M V=P Y$.

Puesto que la oferta monetaria es constante, lo mismo que la velocidad del dinero, un mayor nivel de precios se asocia con un nivel menor de producción real. Dado que $V$ es constante, el valor nominal de todas las transacciones económicas está determinado por la oferta de dinero. Cuando el nivel de precios es mayor, cada transacción requiere más unidades monetarias y por tanto, disminuye la cantidad de bienes y servicios que pueden comprarse. Como la demanda agregada está dada para una cantidad de dinero también dada, un aumento de la oferta monetaria desplaza esta curva a la derecha, si el salario nominal es $W_{0}$, con un nivel de precios mayor, se reduce el salario real.

Asumiendo que los valores de equilibrio en el modelo clásico con una oferta de dinero $M_{0}$ son $Y_{0}, \frac{W_{0}}{P_{0}}$ y $L_{0}$, al aumentar la cantidad de dinero por la vía del aumento de la oferta monetaria resulta totalmente inefectiva, pues no aumentan ni la renta ni el nivel de empleo. La política implica un desequilibrio en el mercado de dinero $M^{s}>M^{d}$, aumenta la demanda por bienes y servicios, pero la producción ya está predeterminada en $Y_{0}$, está restringido al nivel de equilibrio de pleno empleo $L_{0}$, luego entonces aumentan los precios. Para un salario nominal dado $W_{0}$, el aumento de los precios implica un descenso del salario real, de manera que crea un desequilibrio en el mercado de trabajo $\left(D_{\mathrm{L}}>D_{\mathrm{S}}\right)$, este exceso de demanda de trabajo se da a un salario real $\frac{W_{0}}{P_{0}}$, de manera que la contratación de las empresas lleva el salario nominal hasta alcanzar un nivel que restaura el salario real en su valor de equilibrio. 
En conclusión, una expansión monetaria aumenta el nivel de precios y los salarios reales como el tipo de interés nominal se incrementa al tiempo que todas las variables reales del sistema no se ven afectadas, es decir, el dinero es neutral. La relación del dinero está dada por la velocidad de circulación del dinero, o el número de veces promedio en que una unidad de dinero se usa para realizar una transacción final y es igual a la renta nominal.

Para 1929 y luego de una década de crecimiento continuo, la economía norteamericana enfrentó la Gran Depresión o período de crisis que durará hasta bien entrados los años 40 y la economía mundial enfrentó el empobrecimiento, el desempleo y la ruina por el colapso del mercado bursátil y la caída de la producción industrial. Tanto las tasas de empleo como de crecimiento económico se derrumbaron, así como se derrumbaron los preceptos básicos de la economía clásica, especialmente aquellos que tienen que ver con la igualdad de la oferta y la demanda, el equilibrio y el poder de la mano invisible para promover el bienestar social.

Si bien proporcionaron una justificación convincente para levantar las barreras al comercio heredadas del periodo mercantilista y consolidar y reglar al mercado. Haciendo esto, ayudaron a establecer firmemente todo el complejo de condiciones políticas, sociales, legales, económicas y culturales que conforman el marco dentro del que, al menos en los países avanzados del mundo, a partir de entonces la vida era para vivirla.

La Gran Depresión se inicia en Estados Unidos alrededor de 1929 y culmina en 1941. Se cree que es la crisis económica más grande que haya soportado Estados Unidos y que se extendió a todos los países en mayor o menor medida; al respecto hay diferentes puntos de vista sobre los factores que desencadenaron el colapso de la economía norteamericana. Uno, especialmente importante fue sin duda el aumento de la producción después del final de la Segunda Guerra Mundial o período de expansión económica excepcional. La demanda de bienes y servicios primero fue violenta y en algún punto, saciadas las necesidades, se creó una gran capacidad instalada sin utilizar mientras que simultáneamente con el crecimiento económico, los precios de las acciones se dispararon y la población en general descubrió el dinero aparentemente fácil. Más y más gente compró las acciones, sin entender nada al respecto. El Jueves Negro, el 24 de octubre de 1929, comenzó la baja de precios de las acciones, que sumieron al mundo en la catástrofe. Esta crisis prolongada, en términos del ciclo de la teoría económica significa también el ocaso de la economía clásica. Un paradigma vigente por más de un siglo, una ortodoxia clásica de un momento a otro fue cuestionado, relegándola a un segundo lugar y se inicia lo que se ha denominado la «revolución keynesiana» levantada bajo unos principios radicalmente diferentes, pero sobre todo coincidentes en algunos aspectos con el pensamiento mercantilista.

Por otra parte, si se construye un orden mundial bajo los supuestos de competencia perfecta y el rechazo a las barreas comerciales y la intervención pública, con la creencia de que el comercio es benéfico para todos los participantes, creencia arraigada en la teoría clásica del comercio internacional, la teoría keynesiana propicia un cambio transcendental en las relaciones interna- 
cionales, por el marcado proteccionismo que supone el modelo, la intervención pública, esencialmente en la conformación de un orden económico y político sustancialmente diferente, determinado por las organizaciones económicas multilaterales como el BM y el FMI y la Organización de las Naciones Unidas en el plano político. La siguiente sección se dedica enteramente a la presentación de la economía keynesiana, para allegar no solo algunas conclusiones, también las implicaciones que tiene tanto para las relaciones internacionales como para la elaboración y puesta en práctica de la política económica que es justamente una manifestación concreta de las ideas keynesianas.

\section{LA ECONOMÍA KEYNESIANA}

Una manifestación de la importancia que cobraron las ideas keynesianas durante este periodo fue la concepción y puesta en práctica del Nuevo Trato (New Deal) por parte de la administración del presidente Roosevelt en Estados Unidos, o conjunto de medidas encaminadas a superar la crisis ocasionada por la Gran Depresión y que básicamente consistieron en un aumento de los impuestos y del gasto público, lo mismo que una planificación económica mayor dado el papel que ahora juega el estado en el manejo de la economía pero que caracteriza al período con economías relativamente más cerradas al comercio internacional comparadas con el modelo clásico y una intervención mayor del gobierno no sólo el manejo y direccionamiento económiCo, también en muchas otras esferas de la vida social, como la política social, la regulación de los procesos productivos y financieros y un ensanchamiento del aparato burocrático, junto con aranceles elevados y restricciones a las exportaciones, pero al mismo tiempo en comparación con el modelo clásico, visibilizando la mano invisible, es decir, mostrando que el papel de lo público puede jugar un papel muy importante en la asignación de los recursos y donde el estado no es un apéndice del mercado; en buena medida bajo su accionar también se generan resultados económicos que en últimas benefician a la sociedad en su conjunto.

De manera que este sistema teórico emergente se construye sobre algunos conceptos fundamentales como el nivel de empleo, las funciones de consumo e inversión, la ley psicológica fundamental, la teoría de la preferencia por liquidez, el concepto de eficiencia marginal del capital, la teoría del multiplicador y la demanda efectiva. Keynes encontró lagunas en el mecanismo de la competencia perfecta que suponía equilibrio económico con pleno empleo y rompió el pilar en pedazos, el capitalismo del laissez-faire llegó a su fin en 1914 (Schumpeter, 1946). Desafía a la ortodoxia existente e inicia un estudio serio de la macroeconomía; «se tomó el tiempo para liberarse de las cadenas de su pasado clásico. La Teoría General es un ejercicio de purificación para Keynes, que finalmente lo lleva a romper con el cordón umbilical clásico» (Sheehan, op. cit.:9).

Para Keynes, la teoría económica clásica formulada sobre el principio de laissez-faire no puede producir el progreso social necesario; por tanto es inevitable desarrollar una teoría que explique 
las casusas de la crisis y proporcione una alternativa al principio del laissez-faire. Al contrario de sus predecesores, enfatizó las políticas activistas del gobierno para promover un mayor nivel de empleo. Su crítica de la economía clásica incluye a Ricardo, J. S. Mill, Marshall, Edgeworth, Pigou y a los economistas neoclásicos, especialmente en relación con la dicotomía clásica, la teoría del tipo de interés, la teoría del desempleo friccional y voluntario que se asumía por los supuestos implícitos en la Ley de Say.

\subsection{La función de oferta agregada}

Esta función relaciona los niveles posibles alternativos agregados de ventas esperados con el número agregado de trabajadores que las empresas racionales (maximizadoras de beneficios) desean contratar con salarios nominales, la productividad del trabajo (Blanchard, 2009) o el estado de la tecnología y el grado promedio de competencia. (Keynes, 1936); (Davidson, op. cit. 2009:44).

Sobre la función de oferta agregada, cada punto representa el producto de las ventas totales que esperan recibir los empresarios racionales y que deben ser lo suficientemente rentables para justificar un nivel dado de trabajo contratado, de manera que la oferta agregada $(Z)$ o las ventas esperadas son una función creciente de la función de empleo $(N)$; si los empresarios contratan trabajadores adicionales, esperarían que los compradores gasten sumas adicionales de dinero para que les sea rentable expandir la producción.

\subsection{La función de demanda agregada}

Esta función juega un papel preponderante en la teoría keynesiana. A diferencia de los economistas clásicos, para Keynes la oferta y la demanda no son coincidentes en todos los niveles de empleo posibles, no se cumple la Ley de Say. Por tanto, la demanda agregada debe analizarse independientemente de las propiedades y características de la función de oferta clásica. Para la teoría clásica racional (consumidores que maximizan la utilidad) la renta se gasta en bienes y servicios manufacturados ${ }^{5}$ pues sólo este tipo de bienes proporcionan utilidad a los perceptores de renta, principio de racionalidad que es incuestionable ya que constituye la microfundamentación de la demanda agregada. La agregación de las micro-demandas de bienes manufacturados siempre será igual a la renta total ganada y gastada en estos bienes. En la teoría clásica cualquier aumento de la oferta de bienes manufacturados incrementa la renta de las personas, pari passu, por lo que todo incremento del ahorro crea una demanda adicional total equivalente para la producción industrial por el supuesto de racionalidad, por el

\footnotetext{
5 Se entiende como bienes y servicios producidos por la industria manufacturera.
} 
supuesto de que las funciones de oferta y demanda agregadas son iguales, dado que prevalece la Ley de Say.

Sin embargo, Keynes sugiere que la Ley de Say no es observable en el mundo real, de manera que no acepta que solo los bienes que produce la industria generan utilidad para los perceptores de renta y en consecuencia, para su teoría de la demanda agregada la utilidad proviene también de bienes no necesariamente producidos de la industria. Los niveles de producción y empleo están determinados por el gasto agregado. Sus componentes son el gasto en consumo de los hogares $(C)$ y el gasto en inversión de las empresas $(I)$, de manera que $E=C+I$.

En el modelo clásico el consumo, el ahorro y la inversión eran funciones del tipo de interés; en Keynes el consumo es endógeno pero depende del nivel de renta. La rentabilidad esperada es un punto importante de la inversión y el tipo de interés es el costo de tomar prestados los recursos, por lo que el nivel de empleo es una función de inversión, que Keynes lo considera como un factor inestable susceptible a amplias y bruscas fluctuaciones, de manera que los niveles de producción y empleo, que dependen de la inversión, tienen una naturaleza inestable, pues las decisiones de inversión se ven afectadas por la incertidumbre respecto al futuro (volatilidad de las expectativas) que implicaría que la rentabilidad del capital sea también inestable. Esta inestabilidad de la inversión introduce fluctuaciones en la producción y el empleo (Campagna, 1974). La incertidumbre de la inversión se ve afectada por oleadas de pesimismo/optimismo sobre la confianza en la economía. Así visto el problema, el tipo de interés no es la única variable que afecta la inversión. El papel de las expectativas de la rentabilidad futura de la inversión puede llegar a ser más importante aunque el tipo de interés enlaza el presente con el futuro.

Del análisis de la función de consumo Keynes desarrolla el concepto de propensión marginal a consumir, crucial para determinar el tamaño del multiplicador, ya que las perturbaciones en la inversión tienen un impacto magnificado sobre la producción agregada. Un aumento del gasto autónomo induce un incremento de la renta agregada.

El tipo de interés en Keynes no está determinado por variables reales, como el ahorro y la productividad marginal del capital; es un fenómeno enteramente monetario determinado por la demanda de dinero (preferencia por liquidez) y la oferta monetaria que es exógena, determinada por la autoridad monetaria. Rechaza la proposición clásica de que el tipo de interés sea una recompensa por posponer consumo presente, y cree que es la recompensa por desprenderse de liquidez o no acaparamiento (de dinero) en un período específico.

La estructura de la teoría keynesiana de la demanda efectiva asume que el gasto agregado determina los niveles de producción y empleo agregados, que pueden estar sometidos a inestabilidad por la naturaleza inconsistente del gasto en inversión por las influencias que sobre ésta tienen la incertidumbre y las expectativas sobre el futuro de la economía, creando una preferencia por la liquidez, de modo que las variaciones del mercado de dinero $\left(M^{d} \neq M^{s}\right)$. 
Afectan tanto el nivel de producción como el empleo. Esto conduce a Keynes a rechazar la proposición clásica de la neutralidad de la cantidad de dinero. Disminuir el tipo de interés puede acelerar el gasto agregado por el aumento de la inversión (se supone una eficiencia marginal mayor del capital) y el consecuente nivel multiplicador sobre el nivel de producción y el empleo.

Si la política de rebajar el tipo de interés resultara inefectiva, por la vía de la modificación al gasto público y los impuestos (política fiscal) también puede estimularse el gasto agregado al aumentar el empleo o la renta disponible, de manera que el gobierno puede afectar directamente la producción y el empleo a través de la política fiscal. El aumento del gasto público en la compra de bienes y servicios al sector productivo, ceteris paribus, aumenta las ventas del sector industrial acrecentando de esa manera el número de trabajadores contratados y en la medida en que el gobierno cobre sus impuestos al sector privado, reduce la renta disponible y reduce la propensión marginal a consumir, por tanto el gasto en consumo.

\subsection{El mercado de trabajo}

El salario nominal, en opinión de Keynes, no se ajusta en el corto plazo para mantener la economía en el pleno empleo, punto de vista expuesto por la economía clásica para quienes la oferta y la demanda de trabajo son funciones del salario real. Al igualarse, determinan el salario real y el nivel de empleo. (los economistas clásicos supusieron que los salarios nominales eran perfectamente flexibles y se ajustan para igualar la oferta y la demanda de trabajo para mantener la economía de pleno empleo). Keynes ataca esta supuesta flexibilidad de los salarios; para él los salarios nominales son rígidos. A los trabajadores les interesa el salario relativo y absoluto si se considera un mercado de trabajo heterogéneo. Con distintas profesiones y habilidades de la fuerza de trabajo es difícil fijar el salario (es relativo) y que al mismo tiempo sea aceptable a las distintas clases de trabajadores. Las diferencias salariales pueden medirse por salarios nominales relativos porque el nivel de precios termina afectando de igual condición todos los salarios, de manera que la oposición de la fuerza de trabajo a la reducción de los salarios nominales, aunque se redujera la demanda de trabajo, se percibe como una imposición, una injusticia en la estructura de salarios relativos. Si los trabajadores de un sector aceptaban una reducción del salario nominal sería como una variación de los salarios relativos; no tenían la seguridad de que los trabajadores de todos los sectores aceptaran este cambio en los salarios nominales, mientras que la disminución del salario real debido al aumento de precios no se interpretaría de esa manera. La caída en los salarios reales debido al incremento del nivel de precios no tendría la misma resistencia por parte del sector laboral. No habría la convicción de cambios en la estructura de los salarios relativos, de manera que la disminución del salario real por el incremento del nivel de precios encontraría menos oposición que un descenso equivalente en el salario real resultante de una reducción del salario nominal. Al respecto ver (Froyen, 1994). 
El otro factor que incide en la rigidez del salario nominal es de orden institucional. Por cuenta de las negociaciones obrero patronales se fijan contratos de trabajo de entre dos y tres años, que no solo fijan el salario nominal para un periodo ídem sino que, de otra parte, no están sujetos a las oscilaciones del mercado de trabajo durante la duración del contrato, que pueden estar indexados, pero de todas maneras los contratos de salarios nominales fijos confieren rigidez al salario nominal, en consecuencia, las empresas contratan trabajo que maximice el beneficio a un salario nominal fijo. Pero aun cuando explícitamente no se firme un contrato, tácitamente se establece el salario nominal por algún período de tiempo, de manera que los empresarios no pueden reducir el salario nominal si se cae la demanda de los bienes producidos y consecuentemente la demanda de trabajo.

«Los acuerdos contractuales son básicos para una comprensión de la forma cómo funcionan los mercados de trabajo modernos. La visión contractual (énfasis en el original) del mercado de trabajo se sitúa en abierto contraste con la visión del mercado de subasta (énfasis en el original) sin fricciones» (op. cit, Froyen: 234) o como lo expresa Okun (citado en Froyen: 234 y ss.); (Okun, 1981) «... los salarios no se fijan para equilibrar los mercados en el corto plazo, sino más bien están fuertemente condicionados por consideraciones de largo plazo que involucran...las relaciones entre patrono y trabajador. Estos factores aíslan los salarios... a un grado significativo a partir del impacto de los desplazamientos en la demanda de manera que debe hacerse un ajuste en el empleo y la producción».

Con un salario nominal fijo en el corto plazo, no existe la posibilidad de que el salario nominal se ajuste instantáneamente para equilibrar el mercado de trabajo. Ahora, analizando la situación en la que el salario nominal es fijo, es necesario ver qué acontece ante un exceso de oferta de trabajo, es decir, de desempleo. Con $\mathrm{W}_{0}=\overline{\mathrm{W}}_{0}$ y $\mathrm{W}^{\mathrm{s}}>\mathrm{W}^{\mathrm{D}}$.

El nivel de empleo se determina por la demanda. La empresa que maximiza beneficios contrata trabajo hasta el punto en que el salario real es igual a la productividad marginal del trabajo, es decir

$$
\frac{W}{P}=P M g_{L} \text {, es decir, } W=P M g_{L} P
$$

el salario nominal es igual al producto del ingreso marginal. Si se da, como se supuso, un exceso de oferta de trabajo y un salario nominal fijo, el nivel de empleo está en función de la demanda de trabajo, entonces, a un salario nominal fijo se genera un nivel de empleo $N_{0}$.

La curva de oferta de trabajo no juega ningún papel. La posición de la curva de demanda de trabajo depende del nivel de precios. La cantidad de trabajadores contratados por las empresas así como el nivel de producción dependen del nivel de precios. Ante un incremento de los precios y un salario nominal fijo incrementa el valor nominal del producto nominal del trabajo $P M g_{L} P$ en cualquier nivel de empleo lo que lleva a un incremento de la demanda de trabajo, se incrementa la producción. Los precios mayores incentiva una mayor oferta de bienes y servicios 
hasta alcanzar el pleno empleo, de manera que incrementos posteriores de los precios no tienen efectos sobre la producción.

Sin embargo, la crisis económica de los años 70, marcada por la inflación, el desempleo y un crecimiento económico errático, hizo estremecer los cimientos de la teoría keynesiana, Ilamada ahora ortodoxia, por la recuperación de varios de los postulados de la economía clásica.

\section{LA REVOLUCIÓN DE CHICAGO}

El monetarismo es una escuela de pensamiento económico que dominó el análisis macroeconómico en la década de los 60. Aunque entre los monetaristas figuran numerosos y destacados economistas, el más notorio es Milton Friedman. Esta escuela constituyó la corriente crítica principal a la escuela keynesiana. Los monetaristas defienden la ecuación cuantitativa del dinero, según la cual la oferta monetaria es el único determinante de la demanda agregada de la economía, suponiendo constante la velocidad de circulación del dinero. Las variaciones en la cantidad de dinero determinan las variaciones en la demanda agregada, que se transmiten a unos mayores niveles de producción o a un mayor nivel de precios, dependiendo de cómo se comporte la oferta agregada.

Para la segunda posguerra se quiso rehacer la disciplina económica a partir del concepto de competencia perfecta y la eficiencia del mercado. El supuesto de mercados competitivos es la esencia del análisis económico monetarista donde se encuentra un retorno a la economía clásica, y desde luego implica el rechazo al papel regulador del gobierno en los resultados económicos. Anticipando a Friedman, es significativo el trabajo de George Stigler quien «utilizó su creciente estatus en los años inmediatos a la posguerra para imponer su visión de la economía (Freedman, 2008).

Acudiendo a los elementos de la filosofía liberal clásica es evidente el intento de redefinir la naturaleza de la economía con base en la eficiencia y la maximización de la libertad definida en términos de la elección económica y separando el poder económico de la esfera política, pues el poder político actúa como una restricción a las elecciones de los agentes económicos y por tanto de su libertad. Se trata de preservar la libertad en presencia de una amenaza constante, básicamente una resistencia concreta a la intervención del gobierno en esferas que no le corresponden.

El análisis económico monetarista es un análisis del equilibrio que proporciona las bases de la distribución de acuerdo con la productividad marginal individual mientras que la intervención pública debe tener como objetivo la remoción de los obstáculos a los mercados competitivos, más que perseguir unos resultados específicos. El enfoque monetarista es un llamado que pugna por el regreso a los valores subyacentes al análisis clásico, y es el mercado el que representa la 
estructura de gobierno que permite a los individuos lograr la meta de un sistema económico que fomente la mejora de sí mismo por parte de cada individuo.

Friedman construye a partir de la modificación de la teoría cuantitativa del dinero en la tradición de Cambridge la teoría de la demanda de dinero, que adopta la forma

$$
M=d\left(P, r_{b}-\frac{1}{r_{b}} \frac{d r_{b}}{d t}, r_{e}+\frac{1}{P} \frac{d P}{d t}-\frac{1}{r_{e}} \frac{d r_{e}}{d t}, \frac{1}{P} \frac{d P}{d P} ; w ; \frac{\Upsilon}{r}, u\right)
$$

Expresión que, en palabras de Patinkin (Patinkin, 1969) es una presentación más elegante de la teoría del portafolio keynesiana o para Rogers, una presentación alternativa de la misma teoría, si bien esta formulación tiene diferencias importantes entre la concepción de Friedman y la teoría del portafolio, particularmente en que éste último no sigue los postulados de Wicksell de comparar el mercado con la tasa natural de interés que liga los sectores real y monetario; por el contrario, utiliza un concepto alternativo de equilibrio a largo plazo que refleja tanto la tradición de Chicago, como el imperativo inductivista de su metodología.

Dado que $r$ es un promedio ponderado de la riqueza invertida en bonos y del rendimiento real de la posesión de activos, $\left(r_{b}, r_{e}\right)$ entonces:

$$
M=f\left(P, r_{b}, r_{e}, \frac{1}{P} \frac{d P}{d t} ; w ; \Upsilon, u\right)
$$

Que es una función de grado uno en $P$ y $\Upsilon$ entonces

$$
\frac{M}{\Upsilon}=f\left(r_{b}, r_{e}, \frac{1}{P} \frac{d P}{d t} ; w ; \frac{P}{\Upsilon}, u\right)
$$

Que se considera una mejora sobre la concepción tradicional de la teoría cuantitativa suponiendo constante la velocidad del dinero aunque los estudios empíricos mostraran lo contrario. Esta especificación de Friedman tiene en cuenta los cambios en la velocidad del dinero, de manera que la velocidad y la demanda de dinero son a ser una función de muy pocas variables:

$$
\Upsilon=V\left(r_{b}, r_{e}, \frac{1}{P} \frac{d P}{d t} ; w ; \frac{\Upsilon}{P}, u\right) M
$$

Esta es una redefinición de la teoría cuantitativa del dinero. Recurriendo al supuesto de la buena aproximación Friedman expresa la renta corriente como una buena aproximación a la renta permanente utilizando un número grande de observaciones de la renta actual. La renta permanente se expresa por:

$$
\Upsilon_{P l}=(1-\lambda) \sum_{i=1}^{n} \lambda^{i} \Upsilon_{l-1} ; 0<\lambda<1
$$


Al utilizar la hipótesis de la renta permanente Friedman explica los movimientos seculares y cíclicos en la velocidad del dinero sin recurrir al tipo de interés, consideración basada en observaciones empíricas y no en consideraciones teóricas, que favorece una relación más directa que si se utilizara el tipo de interés, que lleva a Friedman a afrontar la dificultad de explicar el proceso de oferta de dinero y establecer si es endógena o exógena y el mecanismo de transmisión; equilibrio monetario que es explicado por la teoría ortodoxa del portafolio, que llevará a determinar si ésta es endógena o exógena.

La función de demanda de dinero, en términos de Friedman y en su forma explícita está dada por:

$$
\log \frac{M}{N P_{p}}=\gamma+\delta \log \left(\frac{\Upsilon_{p}}{N P_{p}}\right)
$$

Que expresa los balances reales permanentes per cápita como una función del ingreso real permanente per cápita y donde $\delta$ es la elasticidad de los balances reales permanentes per cápita con respecto al ingreso permanente, que resulta ser, según las estimaciones, supremamente elástica.

La inclusión de la hipótesis de la renta disponible en el modelo IS-LM recalca la distinción entre tipos de interés real y nominal; analiza la tasa natural de desempleo y la curva de Philips a largo plazo y en última instancia bosqueja un análisis dinámico para explicar el rebasamiento de la posición de equilibrio final por la renta nominal y los precios. En opinión de Rogers, este «es un marco teórico más bien limitado a la teoría cuantitativa del dinero tradicional» (Rogers, 1991).

Este marco teórico se inscribe en el marco de la concepción neoclásica de Chicago, según la cual los agentes económicos se comportan de manera que el proceso de asignación de recursos genera un resultado que resulta ser óptimo en el sentido de Pareto. Se da como un hecho que la teoría neoclásica es una buena aproximación al comportamiento estable de una economía de mercado en la realidad donde el principio de equilibrio anterior estrecho viene a ser muy importante. Si no hay evidencia de que ocurra lo contrario, se impone el criterio de la buena aproximación: los precios y cantidades corrientes son una buena aproximación a sus valores competitivos de largo plazo, si bien el supuesto de la buena aproximación no se somete a prueba directamente (Reder, 1982) citado en Rogers, op. cit:139.

La estructura metodológica Friedman se erige sobre los imperativos de inductivismo, convencionalismo e instrumentalismo. El inductivismo se basa en la creencia de que la veracidad de una teoría se deriva de la aplicación de la lógica inductiva a enunciados y, a partir de allí derivar enunciados (teorías) generales por inducción. Reconocen la existencia de afirmaciones (enunciados) positivos y normativos, siendo sólo las primeras consideradas verdaderas y por tanto útiles en la construcción teórica. El convencionalismo permite a Friedman enfatizar en la función organizativa de las teorías y el criterio de simplicidad en su selección. De otra parte, el propósito 
de le ciencia económica es para Friedman su capacidad de poder hacer predicciones aunque no se enfatiza lo mismo sobre el papel que juega el realismo de los supuestos.

A este imperativo se le ha llamado instrumentalismo ingenuo, pues considera que la teoría es sólo un instrumento para hacer predicciones sobre el estado de la economía, en consecuencia, ni la teoría ni los términos teóricos que contiene se consideran verdaderos o falsos, lo cual no deja de ser contradictorio, ya que desde la filosofía realista de la ciencia, el realismo hace referencia al contenido verdadero de la teoría. Si Friedman adopta este elemento metodológico, este realismo no lo aplica a los supuestos sobre los que basa su teoría. El interés es manifiesto en el contenido empírico de las predicciones pero no con el realismo o la condición general de los supuestos, porque paradójicamente no están sometidos a prueba.

En opinión de Friedman (Friedman, 1968) Keynes y sus seguidores estaban convencidos de la ineficacia de la política monetaria para lidiar con la crisis económica si la preferencia por la liquidez era absoluta, la alternativa por supuesto era la política fiscal.

\section{CONCLUSIONES}

La teoría económica está cambiando constantemente, y esos cambios son el fruto del desempeño de la economía. A lo largo de estas páginas se ha mostrado que al tiempo que se producen agudas crisis económicas, se produce asimismo la crisis de una teoría que se reemplaza por otra estructura. La lógica que se ha impuesto a través de los tiempos es ortodoxia-crisis-expansiónnueva teoría, pero los avances teóricos, nada desdeñables en el caso de la economía no son enteramente nuevos. Y como se afirmó más arriba, los paradigmas se han establecido en torno al papel del estado en la economía, intervención contra no intervención, a la libertad del mercado y el grado de apertura de una economía doméstica al resto del mundo. Y es así como se han sucedido el mercantilismo intervencionista, la fisiocracia con sus limitaciones obvias pero que brindan bases a la economía clásica y la no intervención estatal y la libertad del individuo como del mercado.

Esta fase fue seguida por la economía keynesiana que afirmaba todo lo contrario: la intervención y la regulación pública a través de la política económica puede coadyuvar en el proceso de asignación de recursos y distribución de la renta, supuestos que van a ser duramente cuestionados por los economistas de Chicago, para quienes las bondades del mercado sin restricciones enaltecen la libertad de elegir del individuo, en un ambiente político bastante conservador. 


\section{BIBLIOGRAFÍA}

- Barro, R. (1977). Long-Term Contracting, Sticky Prices, and Monetary Policies. Journal of Economic Perspectives(3), 305-316.

- Basu, S. (1996). Procyclical Productivity, Increasing Returns or Cyclical Utilization? Quarterly Journal of Economics(111), 719-751.

- Bentham, J. (2007). An Introduction to the Principles of Morals and Legislation. New York: Evergreen Review Inc.

- Blanchard, O. (2009). Macroeconomía (Cuarta Edición ed.). Madrid.

- Bloomfield, A. I. (1938). The Foreign-Trade Doctrines of the Physiocrats

- $\quad$ The American Economic Review, Vol. 28(No. 4 ), pp. 716-735.

- Campagna, A. (1974). Macroeconomics, Theory and Policy. Boston: Houghton Mifflin.

- David Backus, P. K. (1992). International Evidence on the Historical Properties of the Business Cycle American Economc Review(82), 864-888.

- Davidson, P. (2009). John Maynard Keynes. Chippenham: Palgrave Macmilla.

- E. Screpanti, S. Z. (1997). Panorama de Historia del Pensamiento Económico. Barcelona: Ariel.

- Freedman, C. F. (2008). Chigago Fundamentalism: Ideology and Methodology in Economics. Singapur: World Scientific Publishing Co.

- Friedman, M. (1968). The Rol of Monetary Policy. American Economc Review, 58(1), 1-17.

- Froyen, R. (1994). Macroeconomía, Teorías y Políticas. Bogotá: MaGraw Hill.

- Hobbes, T. (2009). Leviathan. Oxford: Oxford University Press.

- Ikeda, Y. (2006). Leon Walras end the English Classical School: Walra's Production Theory Revisited. In J. G. Backhaus (Ed.), From Walras to Pareto (pp. 37-48). New York: Springer.

- Keynes, J. M. (1936). The General Theory of Employment, Interest and Money. New York: Harcourt, Brace.

- Locke, J. (2003). Two Treatises on Government and a Letter Concerning Toleration. New Haven: Yale University Press.

- Marshall, A. (2009). Principles of Economics: Cosimo Classics; 8 Una Edition.

- Menger, C. (2007). Principles of Economics. 
- $\quad$ Mill, J. S. (2004). Amherst: Prometeus Books.

- Mill, J. S. (2009). Utilitarianism. Adelaide: eBooks@adelaide.

- Mills, J. (2003). A Critical History of Economics. Londres: Palgrave-Macmillan.

- Okun, A. (1981). Prices and Quantities. Washington: The Brookings Institution.

- Patinkin, D. (1969). The Chicago Tradition, The Quantity Theory and Friedman. Journal of Money, Credit and Banking(1), 56-70.

- $\quad$ Pigou, A. C. (2010). The Economics of Welfare: General Books, LLC.

- Quesnay, F. (1758 (1972)). Le Tableau Économic. New York: Mcmillan.

- $\quad$ Reder, M. W. (1982). Chicago Economics: Permanence and Change. Journal of Economic Literature(20), $1-38$.

- $\quad$ Ricardo, D. (2010). Principles of Political Economy and Taxation: CreateSpace.

- $\quad$ Robert Lucas, J. (1977). Understanding Business Cycle. In A. H. M. K Brunner (Ed.), Stabilization of the Domestic and International Economy: Carnegie-Rochester Conference Series on Public Policy.

- $\quad$ Rogers, C. (1991). Money, Interest and Capital: A Study in the Foundations of Monetary Theory. Cambridge: Cambridge University Press.

- Say, J. B. (1803). Traite d'economie politique, ou simple exposition de la maniere dont se forment, se distribuent, et se composent les richesses, 1803. Paris.

- $\quad$ Schumpeter, J. A. (1946). John Maynard Keynes 1883-1946

- $\quad$ The American Economic Review, Vol. 36(No. 4), pp. 495-518.

- $\quad$ Sheehan, B. (2009). Understanding Keynes' General Theory. Chippenham.

- Shumpeter, J. A. (1995). Historia del Análisis Económico. Barcelona: Ariel.

- Smith, A. (1776). An Inquiry into the Nature and Causes of the Wealth of Nations. London: Methuen and Co., Ltd.

- Viner, J. (1936). Mr. Keynes on the Causes of Unemployment. The Quarterly Journal of Economics, 51(1), 147-167.

- Walras, L. (2003). Elements of Pure Economics. New York: Routledge.

- Wen, J. B. Y. (2003). Indeterminacy, Aggregate Demand, and the Real Business Cycle. Journal of Monetary Economics (51), 503-530. 